\section{Signal transduction pathway involved in platelet activation in immune thrombotic thrombocytopenia after COVID-19 vaccination}

The severe acute respiratory syndrome-coronarovirus2 (SARS-CoV-2) infection is currently advancing and is exponentially developing the COVID-19 pandemic, ${ }^{1}$ especially in the USA, Europe, South America, Russia, and India, recording more than $3,000,000$ deaths. The outbreak of diseases has significantly impacted the lives of millions of people and within a year, several vaccines have been developed to control the pandemia. The European Medicines Agency (EMA), on the basis of randomized, blinded, controlled trials, validated four different vaccines, among them ChAdOx1 nCov-19 (AstraZeneca), a recombinant chimpanzee adenoviral vector encoding the spike glycoprotein of SARS-CoV-2.

The condition of great world emergency has imposed very tight times for the experimentation and controlled trials of these vaccines. Since many people are vaccinated and follow-up is extensive, it might even be possible that new vaccine-related adverse events will arise.

Recently, four studies published by New England Journal of Medicine ${ }^{2-5}$ have described a syndrome characterized by thrombosis and thrombocytopenia that came up 5 to 24 days after initial vaccination with ChAdOx1 nCoV-19 (AstraZeneca). Patients described in the studies were healthy or in medically-stable condition without any previous history of thromboses. Notably, a high percentage of the patients had thrombosis at unusual sites in particular at cerebral venous sinus with a median platelet count at diagnosis of approximately 20,000 to 30,000 per cubic millimeter. ${ }^{4-6}$

In addition to the signs and symptoms of the syndrome and the post-vaccination concomitance, the interesting fact that correlates the individual cases with each other is the high level of antibodies to platelet factor 4 (PF4)polyanion complexes. These are platelet-activating antibodies which clinically mimic autoimmune heparininduced thrombocytopenia (HIT). Indeed, in some heparin-treated patients, the drug combines with a protein produced by platelets, PF4, to form a complex. The binding of the anti-heparin/PF4 antibody to the heparinPF4 complex activates the platelets, leading to their aggregation and thrombocytopenia. ${ }^{7}$ However, unlike the usual situation in HIT, these vaccinated patients did not receive any heparin to explain the later onset of thrombosis and thrombocytopenia.

So far there are very few hypotheses on the pathophysiological mechanisms of post-vaccination PF4-polyanion antibodies. $^{5}$

Some studies described the occurrence of antiphospholipid antibodies ( $\mathrm{aPL}$ ) in some patients vaccinated with ChAdOx1 nCoV-19, ${ }^{3,6}$ suggesting that a wider spectrum of antibodies may play a role in the pathogenesis of vaccine-induced immune thrombotic thrombocytopenia (VITT). Some of the authors have described two cases of malignant middle cerebral artery (MCA) infarction with a concomitant thrombocytopenia within 10 days after vaccination with ChAdOx1 nCoV-19. ${ }^{8}$

Thus, in the present study we analyze in these two patients antibody specificity and the signaling transduction pathway involved in platelet activation in detail.

At the laboratory of the Autoimmunity Unit of the Umberto I Polyclinic of Rome (UTN Unit), we processed sera from two patients (female, age 57 and 55 years, respectively) with VITT, admitted to the UTN Unit, and a serum from a healthy donor vaccinated with $\mathrm{ChAdOx} 1$ nCoV-19 (non-hospitalized female subject, age 52 years). We obtained immunoglobulin $\mathrm{G}(\mathrm{IgG})$ fractions by using protein G-Sepharose columns. Patient 1 revealed right middle cerebral artery occlusion and severe thrombocytopenia $\left(44,000 / \mathrm{mm}^{3}\right)$; patient 2 showed occlusion of the right internal carotid artery terminus and of the left middle cerebral artery, with mild thrombocytopenia $\left(133,000 / \mathrm{mm}^{3}\right)$. Both patients had extensive lung and portal vein thrombosis.

The study was conducted in compliance with the Declaration of Helsinki and the local Ethical Committee approved this study (clinicaltrials gov. Identifier: NCT04844632). For blood samples, healthy donors and relatives of both patients gave written informed consent.

IgG fractions were used to stimulate platelets isolated from healthy donors (females, age range, 36-40 years), who gave written informed consent. Blood samples, in the presence of sodium citrate as anticoagulant, were centrifuged at $150 \mathrm{~g}$ for 15 minutes (min) at $20^{\circ} \mathrm{C}$ to obtain platelet-rich plasma (PRP). Two-thirds of the PRP were drawn, without disturbing the buffy coat layer, in order to prevent contamination. PRP was then mixed with ACD to avoid platelet activation, and centrifuged at $900 \mathrm{~g}$ for $10 \mathrm{~min}$ at $20^{\circ} \mathrm{C}$. Platelet-poor plasma (PPP) was discarded and platelet pellets were resuspended with calcium-free Tyrode's buffer, containing 10\% (v:v) ACD and washed as above. Then, platelets were resuspended in calcium-free Tyrode's buffer with the addition of bovine serum albumin (BSA, $3 \mathrm{mg} / \mathrm{mL}$ ). The purity of the isolated platelets was verified by staining with a fluorescein isothiocyanate (FITC)-conjugated anti-CD61 monoclonal antibody (mAb) (Beckman Coulter, Hialeah, FL, USA) and analyzed by flow cytometry (CytoFLEX, Beckman Coulter), as shown in the Online Supplementary Figure S1A.

Since the p38 mitogen-activated protein kinase (MAPK) pathway is an important intracellular signaling pathway in platelets which can be activated by various stimuli and may be an integral component of arterial and venous thrombosis, ${ }^{9}$ we analyzed by western blot the effect of IgG fractions from these patients on ERK and p38 phosphorylation in platelet lysates.

For this purpose, human platelets, untreated or treated with healthy donor IgG or patient IgG fractions, for 10 min at $37^{\circ} \mathrm{C}$, were resuspended in lysis buffer containing 20 mM HEPES, pH 7.2, 1\% Nonidet P-40, 10\% glycerol, $50 \mathrm{mM} \mathrm{NaF}$ and $1 \mathrm{mM} \mathrm{Na} \mathrm{NO}_{4}$, including protease inhibitors. Then, whole extracts proteins (40 $\mathrm{\mu g} / \mathrm{sample})$ were separated in $10 \%$ SDS-PAGE. Proteins were electrophoretically transferred to PVDF membranes (Bio-Rad Laboratories, Richmond, CA, USA) and then, after blocking with Tris-buffered saline Tween 20 (TBS-T) 3\% BSA, incubated with polyclonal rabbit anti-phospho-ERK1/2 (Cell Signaling, Inc., Danvers, MA, USA), polyclonal rabbit anti-phospho-p38 antibodies (Cell Signaling, Inc.) Antibody reactions were visualized by horseradish peroxidase (HRP)-conjugated anti-rabbit IgG (Sigma-Aldrich, Milan, Italy), and then by the chemiluminescence reaction, using ehnanced chemiluminescence western blot system (Amersham Pharmacia Biotech, Buckinghamshire, UK). In parallel experiments, human platelets were treated with healthy donor IgG or patient IgG fractions for 4 hours at $37^{\circ} \mathrm{C}$, pretreated with ERK inhibitor PD98059 (10 $\mu \mathrm{M}, 3 \mathrm{~min}$ at $\left.37^{\circ} \mathrm{C}\right)$. Then, platelet samples were lysed and analyzed in western blot as above using rabbit anti-tissue factor (TF) $\mathrm{mAb}$ (ab228968,Abcam, Cambridge, UK). Data obtained are expressed as means \pm standard deviation (SD) of at least 
A

B
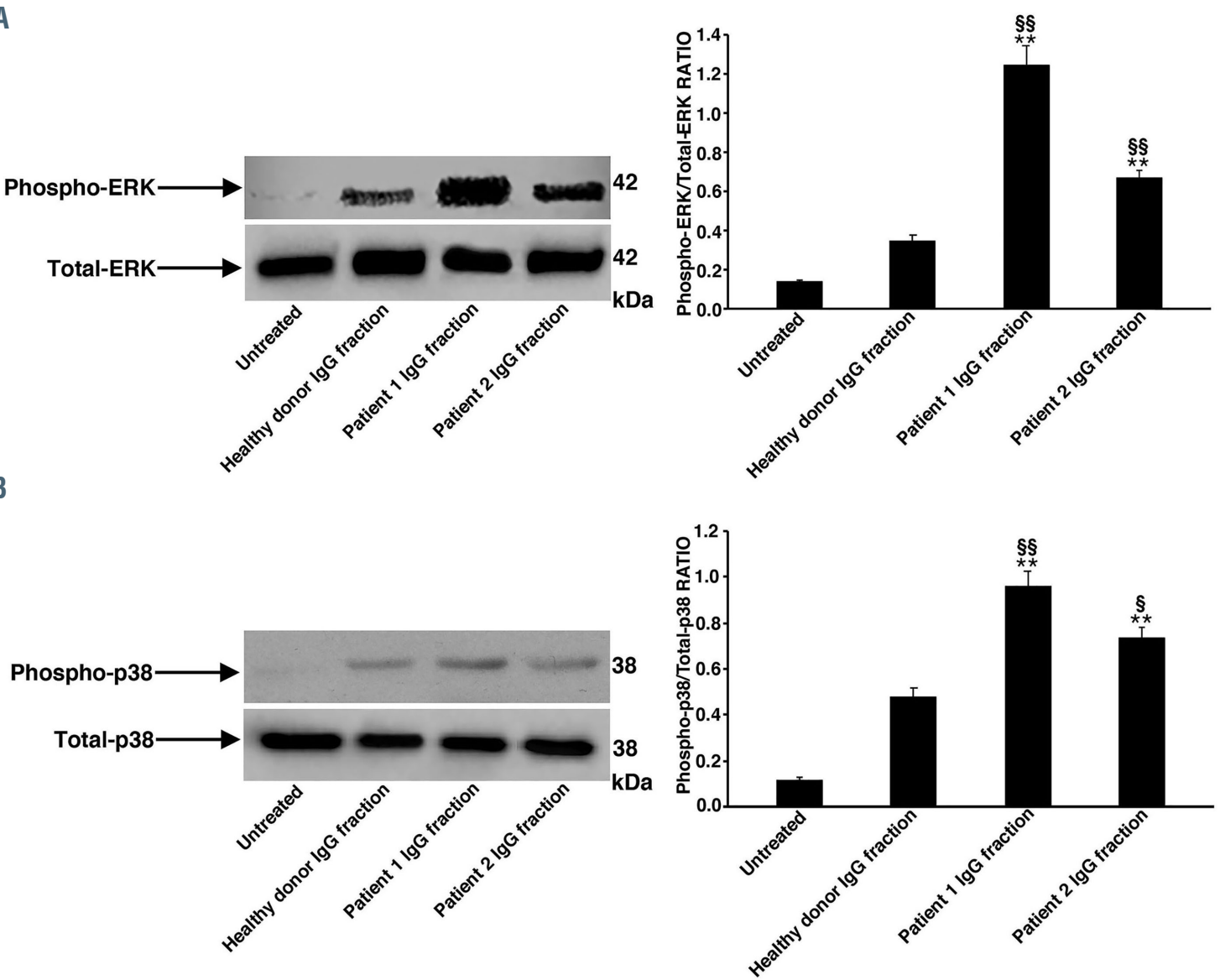

C
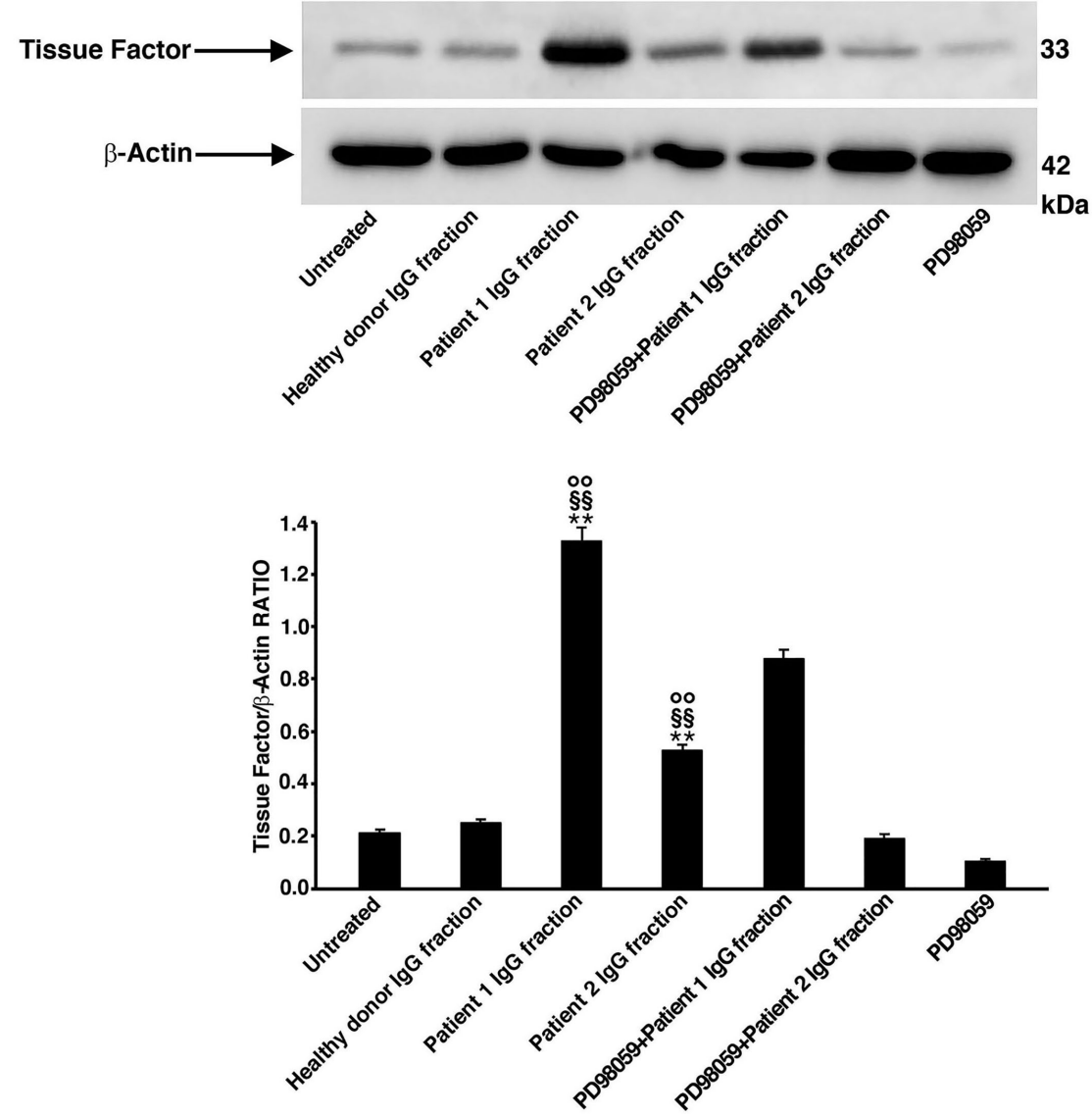

Figure 1. Immunoglobulin G fractions from patients induce ERK and p38 phosphorylation with tissue factor expression. Human platelets from healthy donors were treated with immunoglobulin $G$ (IgG) fractions from the 2 patients and from a healthy donor vaccinated with ChAdOx1 nCoV-19 (10 minutes for ERK and p38 activation and 4 hours for tissue factor [TF] analysis). Protein extracts were separated by SDS PAGE and analyzed by western lot to investigate phosphorylated ERK (A), p38 MAPK (B), and TF (C), using anti-phospho-ERK1/2 anti-phospho-p38 and anti-TF antibodies, respectively. Data are reported as mean \pm standard deviation from 3 independent experiments. Statistical analysis indicated: $\quad * * P<0.001 \quad v s$. untreated; ${ }^{\S \S} P<0.001$ vs. healthy donor IgG fraction; ${ }^{\S} P=0.01$ vs. healthy donor IgG fraction; $P<0.001$ vs. pretreated with PD98059. 
Table 1. Serological characteristics of the patients

\begin{tabular}{|c|c|c|c|}
\hline & Healthy donor & Patient 1 & Patient 2 \\
\hline aPF4 & Negative & Positive $(1,680 \mathrm{AU})$ & Positive (1,290 AU) \\
\hline aCL IgG (GPL/mL) & 0 & 10.4 & 0 \\
\hline aCL IgM (MPL/mL) & 0 & 0 & 0 \\
\hline a 2-GPI IgG (UA/mL) & 0 & 2.9 & 0 \\
\hline a 2-GPI IgM (UA/mL) & 0 & 3.2 & 0.7 \\
\hline LA & 0.95 & 1.8 & 1.4 \\
\hline \multicolumn{4}{|l|}{$\mathrm{aCL}$} \\
\hline (TLC Immunostaining) & Negative & Positive & Positive \\
\hline $\mathrm{aVim} / \mathrm{CL}$ & Negative & Positive & Positive \\
\hline
\end{tabular}

aPF4: anti-platelet factor 4 antibodies; aCL: anti-cardiolipin antibodies; a 2-GPI: anti- 2 glycoprotein I antibodies; LA: Lupus anticoagulant; TLC: thin-layer chromatography; aVim/CL: anti-Vimentin/Cardiolipin complex antibodies.

three independent experiments. Statistical analysis was performed by the paired Student's $t$-test. Statistical significance was set up at $P \leq 0.01$.

Results show that the treatment with both IgG fractions from patients with VITT, induced a significant increase of both phospho-ERK (Figure 1A) and phosphop38 expression (Figure 1B) compared to untreated platelets or treated with healthy donor IgG obtained from a representative healthy donor vaccinated with $\mathrm{ChAdOx} 1 \mathrm{nCoV}-19$. As a consequence of ERK activation, we also observed a significant increase of TF levels in treated platelets. Moreover, pretreatment with ERK inhibitor PD98059 partially prevented TF expression in samples stimulated with patient IgG fractions (Figure $1 \mathrm{C})$, indicating a functional link between ERK activation and TF expression.

Surface TF expression was also verified by flow cytometry analysis, which revealed an increase of TF in platelets treated with IgG fractions from the two patients as compared to healthy donor IgG fraction (Online Supplementary Figure S1B).

Sera of patients and healthy donor were tested for antibodies to platelet factor 4 (PF4)/polyanion by using a commercial enzyme immunoassay (Immucor, Lifecodes, Waukesha, WI). Sera with an optical density $>500$ arbitrary units (AU) were considered as positive. ${ }^{3}$ In addition, anticardiolipin (aCL, IgG, IgM) and anti- $\beta 2$-glycoprotein I ( $\beta 2-\mathrm{GPI}, \mathrm{IgG}, \mathrm{IgM})$ were detected by chemiluminescence assay, using Zenit RA Immunoanalyzer (A. Menarini Diagnostics, Florence, Italy). Finally, aCL were also detected by TLC immunostaining and anti-vimentin/cardiolipin (aVim/CL) antibodies by ezyme-linked immunosorbant assay, as previously described; ${ }^{10}$ lupus anticoagulant (LA) was analyzed by two coagulation systems, a dilute sensitized activated partial thromboplastin time (aPTT) and a dilute Russell's viper venom time (dRVVT), also performing a confirm test (Hemoliance Instrumentation Laboratory, Lexington, MA, USA). Serological characteristics of the two patients and healthy donor are summarized in Table 1.

Previous studies described that a pathogenic PF4dependent syndrome, unrelated to the use of heparin therapy, can occur after the administration of the ChAdOx1 nCoV-19 vaccine. ${ }^{2,3,4-6}$ All subjects displayed anti-PF4 antibodies which were able to activate platelets obtained from healthy donors. ${ }^{2}$ A subset of these antibodies may activate platelets after binding to PF4/heparin complexes, causing the prothrombotic adverse drug reaction HIT. In autoimmune-HIT, anti-PF4/P-antibodies activate platelets in the absence of heparin. In the present study we observed for the first time that IgG fractions of these patients are able to trigger a signal transduction pathway involving ERK and p38 MAPK, which may lead to TF expression increase with a consequent amplification of platelet activation and coagulation cascade. ${ }^{11} \mathrm{We}$ cannot exclude the possibility that other additional signal transduction pathways may be involved.

However, other autoantibody specificities have been described in sera of patients affected by VITT, including $\mathrm{aPL}$ and/or LA. ${ }^{2,4}$ This finding may be relevant, since anti$\beta 2 \mathrm{GPI} / \beta 2 \mathrm{GPI}$ complexes were shown to induce signal transduction pathway(s) leading to platelet activation, ${ }^{12}$ particularly promoting thrombosis via p38 $\mathrm{MAPK}^{13}$ and consequent involvement of TF as major initiator of the clotting cascade. We confirmed and extended the analysis of aPL, also showing the presence of "unconventional" ("non-criteria") aPL in serum of our patients. ${ }^{14}$

How the pathogenic mechanism may be consequent to ChAdOx1 nCov-19 vaccination remains unclear; ${ }^{15}$ however, a possible pathogenic role of the adenoviral viral vector cannot be excluded.

Roberta Misasi, ${ }^{1}$ Antonella Capozzi, ${ }^{1}$ Gloria Riitano, ${ }^{1}$ Serena Recalchi, ${ }^{1}$ Valeria Manganelli, ${ }^{1}$ Vincenzo Mattei, Agostina Longo, ${ }^{1}$ Manuela De Michele, ${ }^{3}$ Tina Garofalo, Fabio M. Pulcinellit and Maurizio Sorice ${ }^{1}$

${ }^{1}$ Department of Experimental Medicine, "Sapienza" University of Rome, Rome; ${ }^{2 B i o m e d i c i n e ~ a n d ~ A d v a n c e d ~ T e c h n o l o g i e s ~ R i e t i ~ C e n t e r, ~}$ Sabina Universitas, Rieti; ${ }^{3}$ Emergency Department, "Sapienza"

University of Rome, Rome, Italy

Correspondence:

MAURIZIO SORICE-maurizio.sorice@uniroma1.it

doi:10.3324/haematol.2021.279729

Received: August 3, 2021

Accepted: September 29, 2021.

Pre-published: October 7, 2021

Disclosures: no conflicts of interest to disclose.

Contributions:FMP and AC designed and performed the research; MDM selected the patients; GR, SR and VM performed experiments; AL and VM provided and analyzed the data; RM and TG wrote the paper; MS designed, organized, and supervised the research and edited the paper. All authors read, edited, participated in the revision, and approved the manuscript.

\section{References}

1. Hu B, Guo H, Zhou P, Shi ZL. Characteristics of SARS-CoV-2 and COVID-19. Nat Rev Microbiol. 2021;19(3):141-154.

2. Scully M, Singh D, Lown R, et al. Pathologic antibodies to platelet 
factor 4 after ChAdOx1 nCoV-19 vaccination. N Engl J Med. 2021;384(23):2202-2211.

3. Greinacher A, Thiele T, Warkentin TE, et al. Thrombotic throm bocytopenia after ChAdOx1 nCov-19 vaccination. N Engl J Med. 2021;384(22):2092-2101

4. Schultz NH, Sørvoll IH, Michelsen AE, et al. Thrombosis and thrombocytopenia after ChAdOx1 $\mathrm{nCoV}-19$ vaccination. $\mathrm{N}$ Engl J Med. 2021;384(22):2124-2130.

5. Cines DB, Bussel JB. SARS-CoV-2 Vaccine-induced immune thrombotic thrombocytopenia. N Engl J Med. 2021;384(23):2254 2256

6. Bayas A, Menacher M, Christ M, et al. Bilateral superior ophthalmic vein thrombosis, ischaemic stroke, and immune thrombocytopenia after ChAdOx1 nCoV-19 vaccination. Lancet. 2021;397(10285):e11.

7. Ahmed I, Majeed A, Powell R. Heparin induced thrombocytopenia: diagnosis and management update. Postgrad Med J. 2007:83(983):575-582.

8. De Michele M, Iacobucci M, Nicolini E, et al. Malignant cerebral infarction, systemic venous thrombosis and thrombocytopenia after ChAdOx1 nCov vaccination: a possible catastrophic varian of vaccine induced thrombotic thrombocytopenia. Nat Commun. 2021;12(1):4663.

9. Adam F, Kauskot A, Rosa JP, et al. Mitogen-activated protein kinas- es in hemostasis and thrombosis. J Thromb Haemost. 2008;6(12): 2007-2016.

10. Truglia S, Mancuso S, Capozzi A, et al. "Non-criteria antiphospholipid antibodies": bridging the gap between seropositive and seronegative Antiphospholipid Syndrome. Rheumatology. 2021 May 10. [Epub ahead of print]

11. Li Z, Zhang G, Feil R, et al. Sequential activation of p38 and ERK pathways by cGMP-dependent protein kinase leading to activation of the platelet integrin alphaIlb beta3. Blood. 2006;107(3):965972.

12. Capozzi A, Manganelli V, Riitano G, et al. Tissue factor overexpression in platelets of patients with anti-phospholipid syndrome: induction role of anti- $\beta 2-\mathrm{GPI}$ antibodies. Clin Exp Immunol. 2019;196(1):59-66.

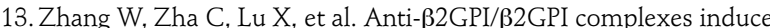
platelet activation and promote thrombosis via p38MAPK: a pathway to targeted therapies. Front Med. 2019;13(6):680-689.

14. Zohoury N, Bertolaccini ML, Rodriguez-Garcia JL, et al. Closing the serological gap in the antiphospholipid syndrome: the value of "non-criteria" antiphospholipid antibodies. J Rheumatol. 2017;44(11):1597-1602

15. Ledford $\mathrm{H}$. How could a COVID vaccine cause blood clots? Scientists race to investigate. Nature. 2021;592(7854):334-335. 